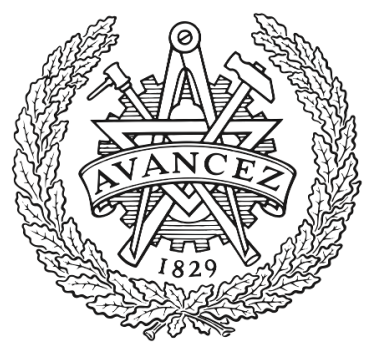

CHALMERS

UNIVERSITY OF TECHNOLOGY

\title{
Strong Transient Flows Generated by Thermoplasmonic Bubble Nucleation
}

Downloaded from: https://research.chalmers.se, 2023-04-26 08:26 UTC

Citation for the original published paper (version of record):

Jones, S., Andrén, D., Antosiewicz, T. et al (2020). Strong Transient Flows Generated by

Thermoplasmonic Bubble Nucleation. ACS Nano, 14(12): 17468-17475.

http://dx.doi.org/10.1021/acsnano.0c07763

N.B. When citing this work, cite the original published paper. 


\title{
Strong Transient Flows Generated by Thermoplasmonic Bubble Nucleation
}

\author{
Steven Jones,* Daniel Andrén, Tomasz J. Antosiewicz, Alexander Stilgoe, Halina Rubinsztein-Dunlop,
} and Mikael Käll*

Cite This: ACS Nano 2020, 14, 17468-17475

Read Online

ACCESS | L W Metrics \& More | 国 Article Recommendations | st Supporting Information

ABSTRACT: The challenge of inducing and controlling localized fluid flows for generic force actuation and for achieving efficient mass transport in microfluidics is key to the development of next-generation miniaturized systems for chemistry and life sciences. Here we demonstrate a methodology for the robust generation and precise quantification of extremely strong flow transients driven by vapor bubble nucleation on spatially isolated plasmonic nanoantennas excited by light. The system is capable of producing peak flow speeds of the order $\mathrm{mm} / \mathrm{s}$ at modulation rates up to $\sim 100 \mathrm{~Hz}$ in water, thus allowing for a variety of high-throughput applications. Analysis of flow dynamics and fluid viscosity dependence indicates that the transient originates in the rapid

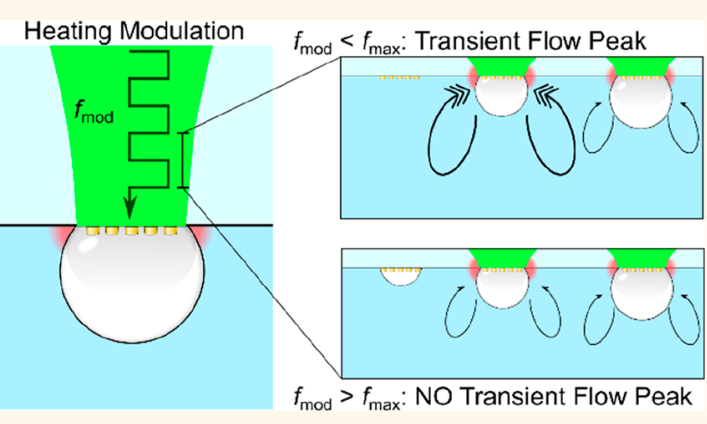
bubble expansion that follows nucleation rather than being strictly thermocapillary in nature.

KEYWORDS: thermoplasmonics, bubbles, Stokeslet, active-manipulation, flow generation

\section{A} chieving efficient liquid transport and mixing at the microscale in spite of the obstacles posed by low Reynolds numbers and laminar flow is integral to microfluidic system development. For instance, detection of analytes at very low concentration remains a key challenge in surface-based biosensing since signal integration times can easily be limited by analyte diffusion. ${ }^{1,2}$ Thus, active transport is typically needed to increase the detection rate so as to reach reasonable assay times. In other applications, e.g., in the development of micromachines and miniaturized engines or motors, the ability to apply large forces mediated by strong localized flows in confined spaces is crucial to or may extend the functionality of such devices. ${ }^{3-5}$

Photothermally induced surface microbubbles show great promise as facile microscopic force and flow actuators since optical control can be achieved through contactless external illumination without the need for any mechanical or electrical interconnects. Bubble generation is typically based on plasmon-enhanced absorption of continuous-wave laser light in semicontinuous metal films to locally superheat water and induce bubble nucleation. ${ }^{6,7}$ The optically established temperature profile around the bubble generates fluid flow via the thermocapillary effect, ${ }^{8}$ resulting in a flow profile well approximated by a Stokeslet near an interface, ${ }^{9}$ and can impart forces over a large volume. ${ }^{10}$ The ability to generate localized fluid flow near interfaces is particularly impactful in microfluidics as this is the region where externally driven flow is least effective at mass transport due to the no-slip condition at the boundary (e.g., Poiseuille flows).

Figure 1a schematically illustrates the current understanding of thermoplasmonic bubble formation and dissipation in water. $6,7,11,12$ The onset of light absorption in the metal near-instantaneously raises its surface-temperature and, for moderate laser powers, causes significant superheating in the surrounding liquid. Beyond a critical local temperature threshold, typically well above the macroscopic boiling point of water, ${ }^{13}$ a bubble can be nucleated via vaporization. Since the surrounding hot fluid is now locally oversaturated, there will be an influx of gas into the bubble causing further growth. This growth stage can continue nearly unimpeded if the heat source is large. After the heating laser is turned off the bubble immediately contracts due to a drop in internal pressure and vapor-condensation. Further bubble dissipation proceeds via expulsion of gas back into the surrounding fluid, a process that is diffusion limited and therefore can be extremely slow for large bubbles. ${ }^{14,15}$

Received: September 15, 2020

Accepted: December 1, 2020

Published: December 8, 2020 


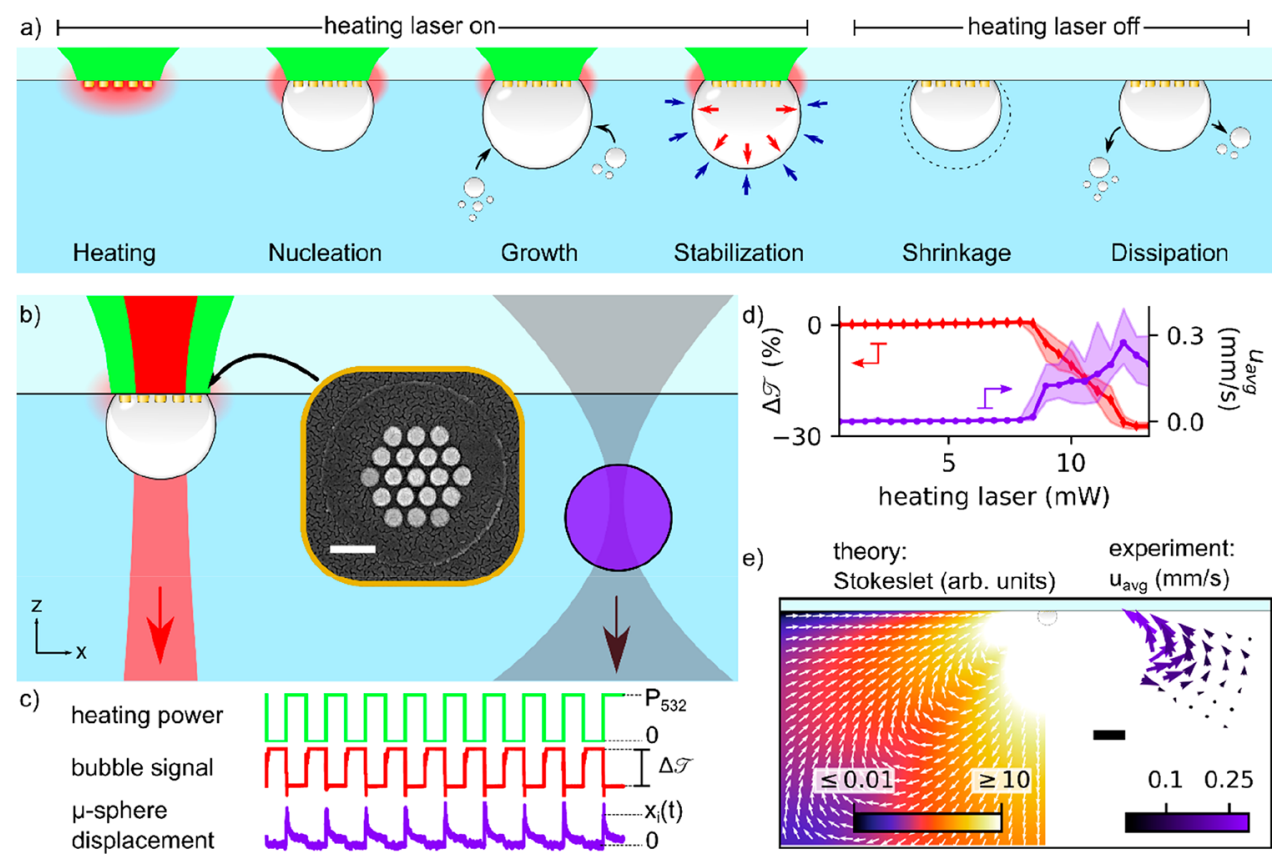

Figure 1. Making bubbles and measuring flows. (a) Overview of the bubble life cycle during heat modulation. (b) Schematic of the experimental environment. (b-inset) Scanning electron micrograph of a nanoantenna (after being used for bubble generation). Scale bar is $300 \mathrm{~nm}$. (c) Experimental procedure showing heating laser modulation (top), changes in detection laser transmission due to bubble formation/dissipation (middle), and probe particle displacement due to bubble induced flow (bottom). (d) Changes in detection laser transmission (left $y$-axis) and local flow (right $y$-axis) for a range of heating laser powers. Data show the average transmission (flow speed) during the on state. Measurement location: $x_{0}, z_{0}=7,-1.5 \mu \mathrm{m}$. Points (shaded region) show the mean (range) from 8 measurements on 7 antennas. (e) Theoretical (left) and experimental (right) flow speed profile in the $x-z$ plane due to the presence of a photothermally generated bubble. Scale bar is $2 \mu \mathrm{m}$. Note that the maximum Reynolds number for the experimental data in (e) is approximately $10^{-3}$.

Recent results on thermoplasmonic bubbles has demonstrated their potential for improving sensing via analyte accumulation, ${ }^{16,17}$ for physical deposition ${ }^{18-20}$ and for chemical synthesis. ${ }^{21,22}$ However, previous works often involve large bubbles that can exhibit semicontinuous growth and require hundreds to thousands of seconds to fully dissipate. ${ }^{6}$ In contrast, for many applications it would be highly advantageous to instead generate small bubbles able to produce strong flows at high modulation rates in order to be utilized in very confined environments and in high throughput systems. The key to such rapid dynamic control of the bubble life cycle is to prohibit the formation of large bubbles to ensure that the gaseous growth stage is quickly terminated. ${ }^{23}$ The transient behavior of bubble formation and dissipation, and the resulting flow response, now become critical for overall system design.

Here, we demonstrate that by utilizing isolated plasmonic nanoantennas for heat generation, it is possible to generate small and quickly dissipating thermoplasmonic bubbles in airequilibrated water. These bubbles are able to produce strong flow transients with flow velocities $>1 \mathrm{~mm} / \mathrm{s}$ several microns away from the bubble at modulation rates up to $\sim 100 \mathrm{~Hz}$ in water, orders of magnitude faster than is possible with larger microbubbles. The bubbles are observed to generate localized Stokeslet-like flow patterns well suited for analyte accumulation, and the persistent flow following the transient is found to be comparable in magnitude (on the order of $100 \mu \mathrm{m} / \mathrm{s}$ ) to those generated previously with larger bubbles. The transient behavior is found to occur from a different driving mechanism (mechanical) than the persistent flow component (thermocapillary). Its dominant character with regard to peak flow-speed provides an avenue for further optimization as a highly advantageous force actuator for integration in next generation microfluidic systems.

\section{RESULTS AND DISCUSSION}

Bubble generation occurs on an isolated nanoantenna (Figure lb-inset) submersed in air-equilibrated water. The thermoplasmonic antenna is heated with a continuous wave $532 \mathrm{~nm}$ laser beam in resonance with the d-band transitions of $\mathrm{Au}$ and the localized dipolar plasmon modes of the structure. This thermoplasmonic antenna configuration was chosen so that the optical and thermal characteristics could be independently controlled by adjusting the size of the individual disks and overall configuration, respectively (further details are given in Thermoplasmonic Antenna: Design and Fabrication). The thermoplasmonic structure utilized here facilitates the generation of small bubbles in air-equilibrated water where bubble growth is quickly terminated by the Laplace pressure and limited volume of heated water. This maintains the bubble radius at approximately $r \approx 500 \mathrm{~nm}$ for the structures used here (depending on heating laser power ${ }^{23}$ ) which enables quick dissipation, after the heating laser is turned off, and therefore high modulation rates. Importantly, the dissipation time for these bubbles, and therefore maximum modulation rate, depends only on the thermoplasmonic antenna size and heating laser power and is not significantly influenced by heating duration.

We use optical force microscopy ${ }^{24}$ in conjunction with optical bubble generation and detection in order to study the transient fluid response during the bubble modulation cycle. Figure $1 \mathrm{~b}$ provides an overview of the measurement procedure (see Supporting Information SI-1 and the Methods section for details of the experimental setup). The heating laser is 
a)

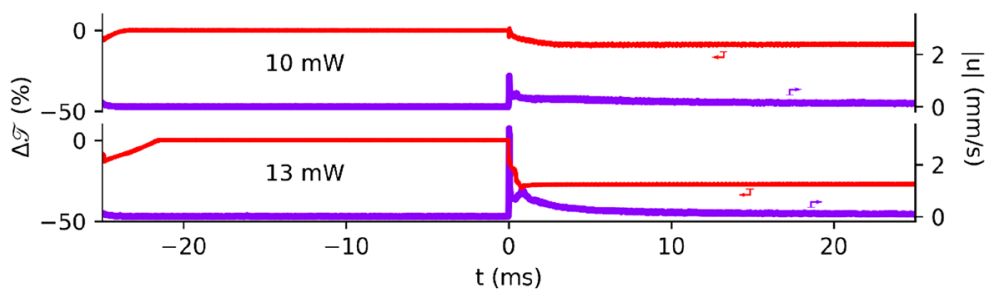

b)

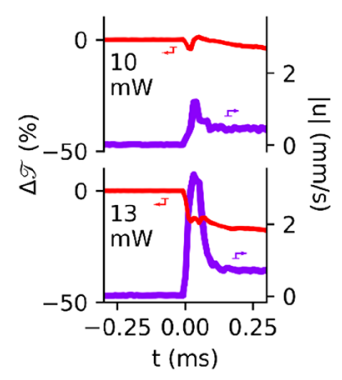

c)

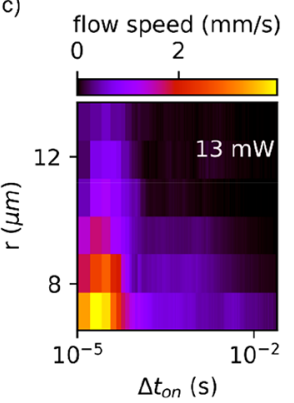

d)

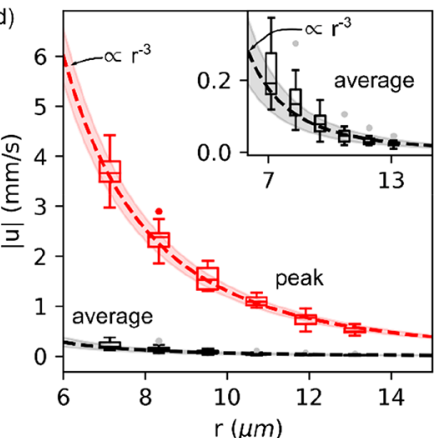

Figure 2. Transient flow dynamics. (a) Full cycle $(20 \mathrm{~Hz})$ trace of the bubble-induced transmission $\left(\Delta \mathcal{T}_{633}\right.$, left $y$-axis $)$ and magnitude of local flow ( $|u|$, right $y$-axis) measured at $x_{0}, z_{0}=7,-1.5 \mu \mathrm{m}$ for heating laser power just above (top) and substantially above (bottom) the bubble nucleation power threshold $\left(P_{532} \approx 8 \mathrm{~mW}\right)$. (b) Zoom-in of transient dynamics at off-to-on transition $(t=0)$. (c) Flow dynamics in logarithmic time scale relative to application of heating laser at increasing radial distance from the antenna $\left(P_{532}=13 \mathrm{~mW}\right) . \Delta t_{\text {on }}$ is the time since the heating laser has been applied in each cycle (all data shown here for $20 \mathrm{~Hz}$ modulation rate). (d) Summary of data from 18 measurements on 6 antennas showing $r^{-3}$ radial decay of both the transient peak flow speed and the average (mean) flow speed. The measurements were performed at locations extending radially from $x_{0}, z_{0}$. Boxes extend from lower to upper quartile values, line indicates median, whiskers show the data range, and outliers are indicated as circles. Note that the Reynolds number for the transient peak is approximately $10^{-2}$.

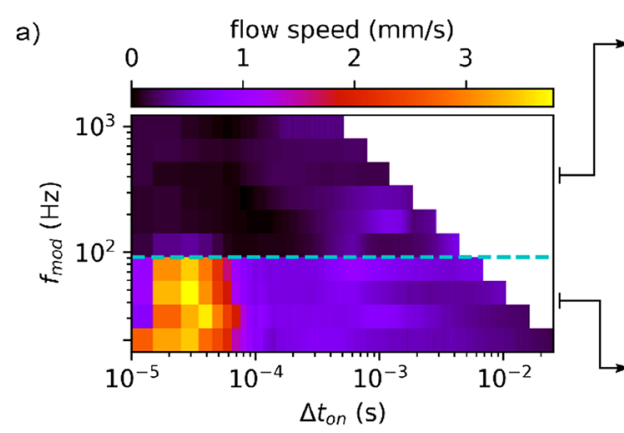

b)
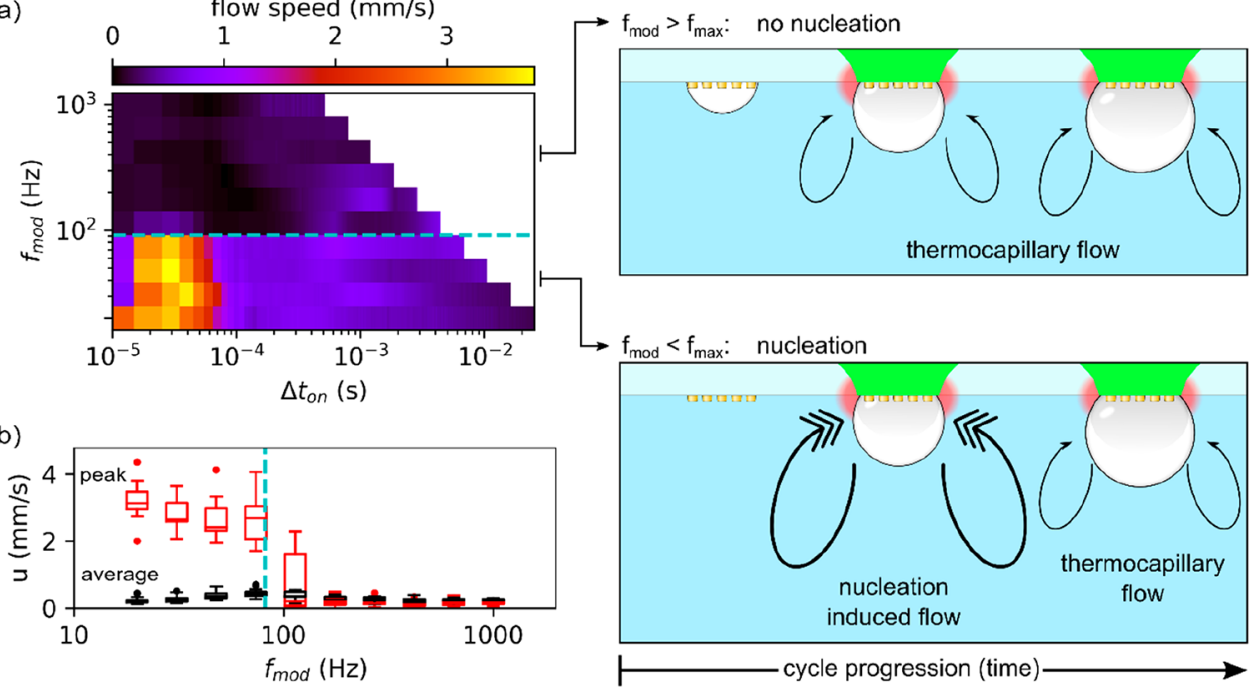

Figure 3. Transient flow is driven by bubble nucleation. (a) Flow dynamics in logarithmic time at various heat modulation frequencies. $\Delta t_{\text {on }}$ is the time since the heating laser has been applied within each heating cycle (for variable heating modulation rates as indicated by the position along the $y$-axis and at a fixed probe location). The dashed blue line indicates the maximum modulation rate $f_{\max }$ for the bubble to fully dissipate before the subsequent heating cycle. The panels to the right indicate the dominant process for $f_{\bmod }<f_{\max }$ (bottom) and $f_{\bmod }>$ $f_{\max }$ (top). (b) Combined data from 17 measurements on 5 nanoantennas showing peak transient and persistent flow speeds at $x_{0}, z_{0}$ versus modulation frequency. Boxes extend from lower to upper quartile values, line indicates median, whiskers show the range, and outliers are indicated as circles. All data shown here were measured for $P_{532} \approx 13 \mathrm{~mW}$ at location $x_{0}, z_{0}=7,-1.5 \mu \mathrm{m}$.

modulated in a square wave temporal profile (duty-cycle $=$ $50 \%$ ) at a preset frequency $f_{\text {mod }}$ and power $P_{532}$ (Figure 1c, upper trace). The bubble is monitored by changes in transmission $\Delta \mathcal{T}_{633}$ of a low power $633 \mathrm{~nm}$ laser beam coaligned with the heating laser since the bubbles are too small and quickly dissipating to be accurately detected with standard brightfield microscopy (Figure 1c, middle trace). Note that
$\Delta \mathcal{T}_{633}$ exhibits a complex variation with bubble radius and contact angle and the signal can therefore not be directly translated to bubble size (see Supporting Information S2b).

Optical force microscopy allows for precise velocimetry of the liquid flow with a high degree of control over the probe location, thus enabling the reconstruction of a 3D flow vector profile with high time resolution. A polystyrene (PS) 
microbead is kept at a variable location $r$ using a precalibrated holographic optical tweezers system operated at $1064 \mathrm{~nm}$, far from the plasmonic resonance. By converting the displacement of the bead (Figure 1c, lower trace) to force $F(r, t)$, where we keep the velocity term from the Langevin equation to increase time-resolution by several orders-of-magnitude (see Supporting Information SI-1 and Supporting Information S4 and S5 for details), we are able to extract the speed of the liquid flow $u(r, t)$ driven by the bubble dynamics with temporal and spatial accuracy far beyond what is possible to achieve using freely diffusing tracer particles. Furthermore, this velocimetry method has the added advantage of avoiding the risk of tracer particles adhering to the bubble, which will perturb the flow profile and the bubble dynamics.

As soon as the heating laser power is increased past the bubble nucleation threshold $\left(P_{532} \approx 8 \mathrm{~mW}\right)$, detected through the change in $\Delta \mathcal{T}_{633}$, we observe strong local flows near the antenna structure (Figure 1d). No measurable flow is observed for powers below this threshold. The experimentally measured average flow profile $\bar{u}(r)$ is highly directional and points toward the nanoantenna at low angles $\phi$ relative to the glass/water interface (Figure 1e right). This flow pattern is well approximated by a Stokeslet near, and perpendicular to, a no-slip boundary (Figure 1e left). Note that the Stokeslet approximation is not specific to any particular driving mechanism but is simply the point-force solution to the Navier-Stokes equation for incompressible flow at a low Reynolds number.

Figure 2 highlights the temporal and radial variation of the flow for $f_{\text {mod }}=20 \mathrm{~Hz}$ (i.e., the duration of the on- and off-states of the heating laser cycle are $25 \mathrm{~ms}$ ). Coinciding with bubble nucleation, signaled by an abrupt drop in $\Delta \mathcal{T}_{633}$, we observe a strong transient flow that ceases within a few hundred microseconds after illumination turns on. The maximum flow speed in the transient, of the order $3-5 \mathrm{~mm} / \mathrm{s}$ at $\phi \approx 12 \mathrm{deg}$ and $r \approx 7 \mu \mathrm{m}$ from the antenna center, is approximately one order of magnitude greater than the subsequent persistent flow (Figure 2a,b).

Radial velocimetry scans (Figure $2 c$ ) reveal that both the transient and the persistent flow components decay proportional to $r^{-3}$ (Figure 2d), as expected for a Stokeslet. Importantly, the overall spatial flow profiles of the transient and the persistent components are nearly identical, i.e., directed toward the antenna at low $\phi$ and from the antenna at higher angles (see Supporting Information S7), and thus, they serve to complement one another for the purpose of mass transport and/or force actuation.

The occurrence of the transient flow peak is intrinsically tied to the act of bubble nucleation. In Figure 3a, we show the bubble dynamics for increasing heating modulation frequency ranging from $f_{\text {mod }}=20 \mathrm{~Hz}$ to $1 \mathrm{kHz}$. It is apparent that a transition occurs at approximately $f_{\max } \approx 100 \mathrm{~Hz}$, above which the transient peak is entirely absent. Importantly, the frequency region above $f_{\max }$ is characterized by a measurable bubble signal $\Delta \mathcal{T}_{633}$ throughout the off-state of the laser heating cycle (see Supporting Information S1c). This implies the bubble does not have time enough to fully dissipate before the start of the next heating cycle. As a result, no nucleation event occurs but instead a small bubble is already present when the heating laser turns on. The dependence of the transient peak on bubble nucleation (i.e., full bubble modulation) was consistently observed as shown in Figure $3 \mathrm{~b}$. In stark contrast, only a marginal effect on the persistent flow speed is observed when passing the transition frequency (see Supporting Information S8a).

From the Stokeslet approximation, one expects that the local flow speed is related to the primary driving force $\mathcal{F}$ as $u \propto \mathcal{F} / \eta$, where $\eta$ is the fluid viscosity. To investigate the mechanism of the transient peak, we measured the flow for several different glycerol-water solutions with varying $\eta$. Again, in all solutions the transient peak is absent for modulation frequencies that do not permit full bubble dissipation (Figure 4a). As seen in Figure 4b, the transient

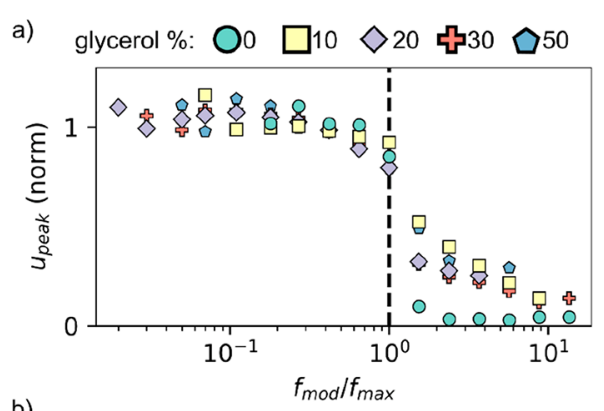

b)

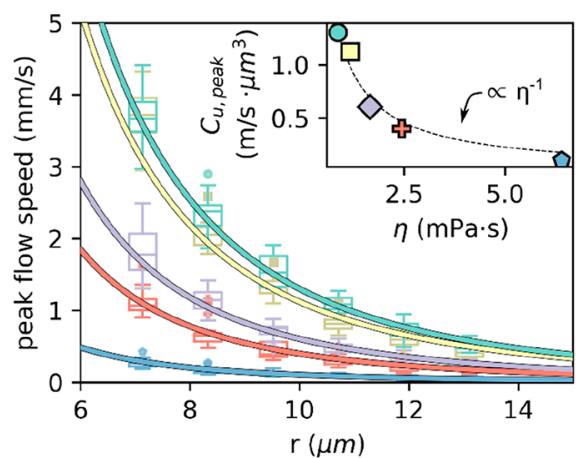

Figure 4. Peak flow is consistent for various water-glycerol solutions. (a) For all solutions there is a significant decline in the transient peak speed when full bubble dissipation cannot occur during the modulation cycle (i.e., $f_{\bmod }>f_{\max }$ ). Note that the maximum modulation rate varies for each solution and all data has therefore been normalized to $f_{\text {max }}$ (b) Peak flow speed radial decay for different solutions. (b-inset) Radial decay fitting coefficient for each solution plotted against the solution viscosity shows a $\eta^{-1}$ dependence. Data in (a) is measured at $P_{532} \approx 13 \mathrm{~mW}$ at location $x_{0}, z_{0}=7,-1.5 \mu \mathrm{m}$ (the same location as data in Figure 3 ) for all solutions; data in (b) is measured at the same locations as the data measured in Figure $2 b, c$. The solution percentages indicate the glycerol content by volume. Data in (a) shows the mean values for several measurements.

radial decay fitting coefficient $C_{u}$ (i.e., $u\left(r, \phi_{0}\right)=C_{u} r^{-3}$ for a specific $\left.\phi_{0}\right)$ shows a well-defined $\eta^{-1}$ behavior. This implies that the primary driving force causing the transient is solution independent for the investigated water-glycerol solutions.

What is the origin of the strong flow transient? We first note that it cannot be a shockwave, which would produce a force propagating strictly outward from the antenna at significantly shorter time scales. Second, the insensitivity to the host fluid (with regards to the driving force) would not be expected if the flow was purely thermocapillary in nature since the thermocapillary effect depends nontrivially on several different material properties (e.g., Henry's constant, interfacial surface tension, and their temperature derivatives, etc.). Indeed, the 
persistent flow component shows no clear quantitative trend between the different solutions (see Supporting Information $\mathrm{S} 8 \mathrm{~b})$.

Based on these observations, we hypothesize that the mechanism responsible for the transient peak is mechanical in nature and qualitatively analogous to the process utilized in thermal inkjet style actuators. ${ }^{23}$ The spectral properties of the plasmonic antenna are nearly identical between solutions (see Supporting Information S9a); thus, approximately the same amount of energy will have been loaded into the system in all cases. When the heating laser power is significantly above the threshold for bubble formation, which is nearly constant for all solutions (see Supporting Information S9b) since the boiling point increases by only $\sim 10 \mathrm{~K}$ up to $50 \%$ glycerol, most of the energy associated with bubble formation will contribute to the expansion of the bubble. Thus, we hypothesize that it is the rapid bubble expansion that mechanically induces the transient fluid flow. Due to the symmetry of the system, this bubble expansion can then be approximated as a point force directed perpendicular to the interface in the Stokeslet picture, which implies that it will result in the same flow profile as the subsequent persistent flow. Spatial integration of the flow profile for realistic bubble sizes and based on the Stokeslet approximation indicates that the work done on the fluid per time unit may correspond to as much as $10 \%$ of the absorbed optical power at the peak of the transient (see Supporting Information section SI-5).

The peak flow speeds/forces achievable during the transient regime of bubble formation provides an opportunity for microscale manipulation. Unlocking the full potential of photothermal surface bubbles for active manipulation in microfluidics requires further understanding and optimization of the transient flow formation. Higher modulation rates are obviously advantageous in applications where the total (cycle averaged) flow speed is the primary figure of merit. The use of smaller heating nanoantennas can enable over an order of magnitude increase in the total modulation rate by heating a smaller volume of water and thus forming smaller bubbles. ${ }^{23}$ However, smaller bubbles would presumably entail lower transient forces and thus the scaling of these two effects should be investigated. Directional control over the flow profile may be achieved by tailoring the antenna shape and/or the surrounding environment to influence the bubble expansion. Optimization of the peak transient force may be achieved by inducing bubble oscillations (and thus possibly inducing oscillating bubble streaming effects) during the initial bubble formation, a phenomenon that has sometimes been observed for larger microbubbles. ${ }^{26,27}$ Other strategies for flow optimization in this system include tailoring the heat source density or utilizing solutal Marangoni effects to control the flow directionality/profile, as has been demonstrated in recent studies on larger microbubbles. ${ }^{28,29}$

\section{CONCLUSION}

In conclusion, we have investigated the formation of flows surrounding thermoplasmonically nucleated bubbles. Specifically, the bubbles studied here are small enough such that the growth is self-limited and thus are suitable for inclusion as actuators in integrated microfluidic platforms. These bubbles have been found to exhibit two distinct flow regimes-an initial transient flow and a subsequent persistent flow. The transient flow was found to be an order of magnitude greater than the persistent flow, while both exhibit the same general flow profile making both features advantageous for a plethora of microfluidic manipulation based applications. Critically, it was found that the transient flow requires nucleation to occur in order to be present, thus highlighting the need for fast bubble modulation to be possible in order to fully utilize the transient phenomenon.

\section{METHODS}

Experimental Setup. A schematic of the experimental setup is shown in Supporting Information section S1a. The general system consists of a heavily modified upright microscope (Nikon Ti80 Eclipse) with a piezo stage (Mad City Laboratories, Nano-LP200) for precision alignment of the antennas and a camera for brightfield imaging used in rough alignment (Andor iXon EMCCD) and a 60X, 1.2 NA water immersion objective (Nikon CFI Plan Apo VC 60XC WI) for laser focusing and imaging. All experimental measurements were automated with LabVIEW. The optical system essentially consists of three main optical paths for the (1) heating laser, (2) bubble detection laser, and (3) optical force microscopy system.

(1) The heating laser used was a $532 \mathrm{~nm}$ continuous wave (CW) laser (Vortran, Stradus 532-40, $532 \mathrm{~nm}$ ) which was chosen to enable strong optical absorption due to spectral overlap with the d-band electronic transition in gold. The maximum heating laser power was controlled by a voltage applied to the control unit, and modulation was achieved with an acousto-optic modulator (Brimrose TEAFI100.4-1.0-MSD-ER). The zeroth order diffraction from the acoustooptic modulator was monitored with a silicon photodiode (Thorlabs, PDA36A-EC), while the first-order diffraction was transmitted to the microscope for photothermal heating. The zeroth order photodiode signal was calibrated to provide a real-time measurement of the heating laser power reaching the sample. For all data shown here, we use square wave heating modulation with a duty-cycle of $50 \%$.

(2) Bubble detection was achieved by a lower power $633 \mathrm{~nm} \mathrm{CW}$ laser (Melles Griot, $\mathrm{HeNe}$ ) which was coaligned with the heating laser. The transmission of this laser was collected with a condenser (Nikon CSI Plan Fluor ELWD 60XC, NA = 0.7, air) and then focused onto a silicon photodiode (Thorlabs PDA36A-EC) to monitor the transmission of the laser for both bubble detection as well as alignment with the structures. A $633 \mathrm{~nm}$ band-pass filter and $1000 \mathrm{~nm}$ short pass filter was placed in front of this photodiode to prevent any leakage from the other lasers when monitoring this signal. A nonlinear response of the transmitted signal with respect to bubble size is expected, as shown in Supporting Information section S1b (calculation details given below); however, this technique can be used to robustly detect the presence of a bubble and thus determine if a bubble is able to fully dissipate as shown Supporting Information section S1c.

(3) The optical force microscopy system consists of a $1064 \mathrm{~nm} \mathrm{CW}$ laser (Cobalt, Rumba 05-01 DPSS) which was expanded to a collimated beam that slightly overfilled the back aperture of the trapping objective. The position of the trapping laser focal spot was controlled by a liquid crystal on silicon spatial light modulator (Meadowlark Optics, $1920 \times 1152$ ) which was placed at a plane conjugate to the back focal plane of the objective in a 4-f configuration. The light transmitted through the trapped particle was collected by the condenser and directed toward a quadrant photodiode (First Sensor, QPD154-Q HVSD, $1064 \mathrm{~nm}$ enhanced). The back focal plane of the condenser was imaged onto the quadrant photodiode so that the output voltage would reflect the wavevector distribution of the transmitted light. A $1064 \mathrm{~nm}$ bandpass filter and several neutral density filters were placed in front of the quadrant photodiode to prevent oversaturation and leakage from the other lasers.

Measurement Procedure. The measurement procedure consists of first trapping the probe particle, positioning the trapped particle at the desired location, and then aligning the heating and bubble detection lasers with the plasmonic antenna. Antenna alignment was performed by measuring the $633 \mathrm{~nm}$ transmission as the antenna was scanned across each axis though the $633 \mathrm{~nm}$ focal spot and then 
moving to the location of lowest transmission; this procedure was automated with LabVIEW. During the measurements, an initial "calibration" time series was obtained (typically $3 \mathrm{~s}$ ) for the antenna aligned and probe particle in the desired position but without application of the heating laser (see Supporting Information section S10 for comparison between experimental data and theoretical limits). After this "calibration" data was collected, the heating laser would then be modulated while data was collected on all detection channels-this consists of the "measurement" time series (typically 2 s). Data was acquired at $100 \mathrm{kHz}$ with a digital acquisition device (DAQ National Instruments, NIUSB6252). Note that both the "calibration" and "measurement" time series were recorded as one series with no break in continuity during data acquisition. After data had been acquired the system would prepare for the next measurement (e.g., change heating laser power, move probe particle, etc.). This type of serial measurement procedure for determining the flow profile is possible because of the high degree of repeatability between measurements as shown in Supporting Information section S3. After a predetermined number of measurements had been obtained the system would automatically realign before proceeding (typically 3 measurement or $\sim 15 \mathrm{~s}$ ). Although some amount of drift was present in our optical system, it was determined that drift could be limited to within $100 \mathrm{~nm}$ along all axes (worst case scenario) if realignment were performed at least once every $120 \mathrm{~s}$. By realigning the system after substantially shorter intervals we were able to essentially eliminate any significant drift from our measurements. The standard deviation (error) of the autoalignment procedure was measured to be $10,12.5$, and $50 \mathrm{~nm}$ along $x, y$, and $z$, respectively.

Further details about the experimental procedure, including trap position calibration, trap force calibration, data analysis, and control experiments, are given in Supporting Information section SI-1.

Thermoplasmonic Antenna: Design and Fabrication. The nominal design of the thermoplasmonic antenna consists of 19 gold disks (height $=60 \mathrm{~nm}$, radius $=50 \mathrm{~nm}$ ) arranged in a hexagonal lattice with interparticle spacing of $150 \mathrm{~nm}$ (center to center) and an overall structure radius of approximately $350 \mathrm{~nm}$. By using a collection of plasmonic disks (as opposed to a single large structure) the optical and thermal properties could be independently controlled by changing size of the individual disk and overall structure diameter, respectively (note that the disks are spaced too far apart to plasmonically couple via near-field coupling and any diffractive orders are in the ultraviolet wavelength region). The thermoplasmonic disks used here were designed such that the plasmonic resonance enabled significant absorption at $532 \mathrm{~nm}$ to facilitate bubble formation via photothermal heating from the heating laser while being nonresonant at the wavelength used for optical force microscopy (1064 nm) although in general there is essentially no interaction with the 1064 $\mathrm{nm}$ laser during the experiments because there is never significant geometric overlap between the trapping laser and the antenna (see SI1, Methods \& Experimental Setup - Control Experiments). The thermal properties of the antenna, dictated by the overall structure size, were designed to enable bubble nucleation at moderate power levels while simultaneously ensuring that the heating was sufficiently localized to prevent continuous bubble growth (hence ensuring high permissible modulation rates). Further details about this thermoplasmonic antenna design, and its optical/thermal properties are given in ref. ${ }^{23}$

The disks were fabricated by electron beam lithography followed by metal deposition and lift-off. Microscope cover slides (\#1.5) were washed under sonication for $5 \mathrm{~min}$ each in acetone and isopropyl alcohol followed by an oxygen plasma treatment $(30 \mathrm{~s}$ at $50 \mathrm{~W}$ and $250 \mathrm{mTorr}$ ). A $150 \mathrm{~nm}$ thick layer of AR-P 6200_13: Anisol, 1:1 resist was deposited via spin-coating and cured at $160^{\circ} \mathrm{C}$. A sacrificial layer of $\mathrm{Cr}$ was deposited to present a reflective and conductive surface for the subsequent electron beam lithography. The nanodisks were exposed in a Raith EBPG $5200100 \mathrm{kV}$ system to a dose of $\approx 533 \mu \mathrm{C} /$ $\mathrm{cm}^{2}$. The $\mathrm{Cr}$ was removed by wet etching and the resist developed in $n$-Amylacetate for $90 \mathrm{~s}$. Metals for the antennas $(2 \mathrm{~nm}$ of $\mathrm{Ti}$ for adhesion and $60 \mathrm{~nm}$ of $\mathrm{Au}$ ) were evaporated and then uncovered via lift-off in Remover 1165. To enhance optical properties and thermal stability, 10 min annealing at $150{ }^{\circ} \mathrm{C}$ followed by plasma enhanced chemical vapor deposition of $20 \mathrm{~nm}$ of $\mathrm{SiO}_{2}$ conclude the fabrication.

Sample Preparation. Before experiments, the coverslips containing the plasmonic antenna were rinsed with isopropanol and deionized water and dried with nitrogen. Any biological contaminants on the surface were then removed by cleaning the sample with air plasma etching (Harrick Plasma, PDC-32G) for $60 \mathrm{~s}$ on high. A silicone spacer (Secure-Seal Spacer, $13 \mathrm{~mm}$ diameter, $120 \mu \mathrm{m}$ depth) was adhered to the sample. The microwell was then filled with a dilute solution containing the probe microspheres (micro particles $\mathrm{GmbH}$, diameter $=1.98 \mu \mathrm{m}$, standard deviation $=0.03 \mu \mathrm{m})$ in either deionized water or a deionized water + glycerol solution (SigmaAldrich G9012-500 ML, Glycerol, 99\%). All solutes are airequilibrated before sample preparation. The sample and filled microwell was then sealed against a standard microscope slide and loaded into the experimental setup. After measurements had finished, the sample was left in an isopropanol bath until it detached from the silicone spacer and cleaned again before storage for further use.

Bubble Detection Simulations. Simulations of the transmitted $633 \mathrm{~nm}$ signal were carried out using the finite-difference timedomain method (FDTD Solutions, Lumerical). The plasmonic nanoantennas were made of gold described by a complex permittivity according to Johnson and Christy ${ }^{30}$ and their size and layout matched the experimental dimensions: hexagonal pattern with a lattice of 150 $\mathrm{nm}$ composed of 19 nanodisks with vertical side walls $60 \mathrm{~nm}$ in thickness, $5 \mathrm{~nm}$ top and bottom edge rounding, and diameter of 95 $\mathrm{nm}$ (slightly smaller than the nominal design radius. They were placed on a substrate with a refractive index of 1.5 and covered by a conformal $20 \mathrm{~nm}$ thick dielectric layer with index of 1.5. The medium on top of the structure is water with $\mathrm{n}=1.33$. Illumination of the structures was from the substrate side with a broad-spectrum plane wave to match the simulated spectrum to the experimental one and a Gaussian beam with a waist radius of $800 \mathrm{~nm}$ to model transmission changes at $633 \mathrm{~nm}$ induced by bubble growth. Permittivity changes with temperature were neglected. The forward scattered signal was collected over a large area $(30 \mu \mathrm{m} \times 30 \mu \mathrm{m}, 2.4 \mu \mathrm{m}$ from the substrate) to perform far-field propagation to account for the collection efficiency of the objective. The bubble was modeled as an air-filled sphere with a variable radius $(100-1000 \mathrm{~nm})$ and a variable contact angle (30,60, 90, 120 degrees). The mesh size around the nanodisks in a volume $800 \mathrm{~nm} \times 700 \mathrm{~nm} \times 130 \mathrm{~nm}$ was set to $2 \mathrm{~nm}$, followed by a gradual mesh increase to $8 \mathrm{~nm}$ and then 12 $\mathrm{nm}$. Symmetric and antisymmetric boundary conditions were also used.

The transmitted signal for all studied contact angles has a similar dependence with bubble size. For small bubbles with radii less than approximately $400 \mathrm{~nm}$ the transmitted signal initially increases. Evidence of this transmission increase for small bubbles can be inferred from S1c where it can be seen that as the bubble dissipates, the transmission briefly increases before bubble dissipation is complete. Once the bubble size exceeds the lateral dimensions of the nanostructure, the signal begins to decrease more quickly than the initial increase. This decrease is nonmonotonic and Fabry-Perot-like oscillations are present. In general, the slope of this decrease in transmitted signal intensity is proportional to the contact angle, which determines the overall bubble size. The slope of the signal dependence is the largest for the $30^{\circ}$ contact angle and decreases ca. 5-fold for the largest studied angle of $120^{\circ}$.

\section{ASSOCIATED CONTENT}

\section{Supporting Information}

The Supporting Information is available free of charge at https://pubs.acs.org/doi/10.1021/acsnano.0c07763.

Further details about experimental setup and data analysis technique; control experiments; $x z$-flow profile for transient peak and persistent flow; modulation rate effect on persistent flow in aqueous glycerol solutions; darkfield spectra of nanoantennas and bubble threshold 
in aqueous glycerol solutions; and details about energy dissipation calculation (PDF)

\section{AUTHOR INFORMATION}

\section{Corresponding Authors}

Mikael Käll - Department of Physics, Chalmers University of Technology, 41296 Gothenburg, Sweden; 이이이.org/00000002-1163-0345; Email: mikael.kall@chalmers.se

Steven Jones - Department of Physics, Chalmers University of Technology, 41296 Gothenburg, Sweden; 이이.org/00000003-2813-1945; Email: steven.jones@chalmers.se

\section{Authors}

Daniel Andrén - Department of Physics, Chalmers University of Technology, 41296 Gothenburg, Sweden; (1) orcid.org/ 0000-0003-0682-5129

Tomasz J. Antosiewicz - Faculty of Physics, University of Warsaw, 02-093 Warsaw, Poland; 이이.org/0000-00032535-4174

Alexander Stilgoe - School of Mathematics and Physics, University of Queensland, Saint Lucia 4072, Queensland, Australia

Halina Rubinsztein-Dunlop - School of Mathematics and Physics, University of Queensland, Saint Lucia 4072,

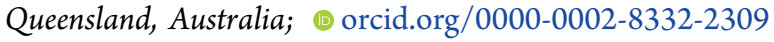

Complete contact information is available at:

https://pubs.acs.org/10.1021/acsnano.0c07763

\section{Author Contributions}

S.J. and M.K. conceived the study. S.J., A.S., and H.R.D. designed the experimental setup. S.J. performed the measurements and analyzed the results. D.A. fabricated the samples. T.J.A. performed optical simulations. S.J. and M.K. interpreted the data and wrote the paper with input from all other authors.

\section{Notes}

The authors declare no competing financial interest.

\section{ACKNOWLEDGMENTS}

This work was funded by the Swedish Research Council and Chalmers Excellence Initiative Nano.

\section{REFERENCES}

(1) Squires, T. M.; Messinger, R. J.; Manalis, S. R. Making It Stick: Convection, Reaction and Diffusion in Surface-Based Biosensors. Nat. Biotechnol. 2008, 26, 417-426.

(2) Sheehan, P. E.; Whitman, L. J. Detection Limits for Nanoscale Biosensors. Nano Lett. 2005, 5, 803-807.

(3) Marmottant, P.; Hilgenfeldt, S. Controlled Vesicle Deformation and Lysis by Single Oscillating Bubbles. Nature 2003, 423, 153-156.

(4) Asavei, T.; Nieminen, T. A.; Heckenberg, N. R.; RubinszteinDunlop, H. Fabrication of Microstructures for Optically Driven Micromachines Using Two-Photon Photopolymerization of UVcuring Resins. J. Opt. A: Pure Appl. Opt. 2009, 11, 034001-034007.

(5) Quinto-Su, P. A. A Microscopic Steam Engine Implemented in an Optical Tweezer. Nat. Commun. 2014, 5, 5889-7.

(6) Baffou, G.; Polleux, J.; Rigneault, H.; Monneret, S. SuperHeating and Micro-Bubble Generation around Plasmonic Nanoparticles under CW Illumination. J. Phys. Chem. C 2014, 118, 48904898.

(7) Wang, Y.; Zaytsev, M. E.; Le The, H.; Eijkel, J. C. T.; Zandvliet, H. J. W.; Zhang, X.; Lohse, D. Vapor and Gas-Bubble Growth Dynamics around Laser-Irradiated, Water-Immersed Plasmonic Nanoparticles. ACS Nano 2017, 11, 2045-2051.
(8) Takahashi, K.; Weng, J.-G.; Tien, C.-L. Marangoni Effect in Microbubble Systems. Microscale Thermophys. Eng. 1999, 3, 169-182.

(9) Blake, J. R.; Chwang, A. T. Fundamental Singularities of Viscous Flow. Part I: The Image Systems in the Vicinity of a Stationary NoSlip Boundary. J. Eng. Math. 1974, 8, 23-29.

(10) Namura, K.; Nakajima, K.; Suzuki, M. Quasi-Stokeslet Induced by Thermoplasmonic Marangoni Effect around a Water Vapor Microbubble. Sci. Rep. 2017, 7, 45776-45778.

(11) Li, X.; Wang, Y.; Zaytsev, M. E.; Lajoinie, G.; Le The, H.; Bomer, J. G.; Eijkel, J. C. T.; Zandvliet, H. J. W.; Zhang, X.; Lohse, D. Plasmonic Bubble Nucleation and Growth in Water: Effect of Dissolved Air. J. Phys. Chem. C 2019, 123, 23586-23593.

(12) Zaytsev, M. E.; Wang, Y.; Zhang, Y.; Lajoinie, G.; Zhang, X.; Prosperetti, A.; Zandvliet, H. J. W.; Lohse, D. Gas-Vapor Interplay in Plasmonic Bubble Shrinkage. J. Phys. Chem. C 2020, 124, 5861-5869.

(13) Baffou, G. Thermoplasmonics, 1st ed.; Cambridge University Press: Cambridge, 2018.

(14) Epstein, P. S.; Plesset, M. S. On the Stability of Gas Bubbles in Liquid-Gas Solutions. J. Chem. Phys. 1950, 18, 1505-1509.

(15) Ljunggren, S.; Eriksson, J. C. The Lifetime of a Colloid-Sized Gas Bubble in Water and the Cause of the Hydrophobic Attraction. Colloids Surf., A 1997, 129-130, 151-155.

(16) Kang, Z.; Chen, J.; Ho, H.-P. Surface-Enhanced Raman Scattering: Via Entrapment of Colloidal Plasmonic Nanocrystals by Laser Generated Microbubbles on Random Gold Nano-Islands. Nanoscale 2016, 8, 10266-10272.

(17) Karim, F.; Vasquez, E. S.; Sun, Y.; Zhao, C. Optothermal Microbubble Assisted Manufacturing of Nanogap-Rich Structures for Active Chemical Sensing. Nanoscale 2019, 11, 20589-20597.

(18) Lin, L.; Peng, X.; Mao, Z.; Li, W.; Yogeesh, M. N.; Rajeeva, B. B.; Perillo, E. P.; Dunn, A. K.; Akinwande, D.; Zheng, Y. Bubble-Pen Lithography. Nano Lett. 2016, 16, 701-708.

(19) Bangalore Rajeeva, B.; Lin, L.; Perillo, E. P.; Peng, X.; Yu, W. W.; Dunn, A. K.; Zheng, Y. High-Resolution Bubble Printing of Quantum Dots. ACS Appl. Mater. Interfaces 2017, 9, 16725-16733.

(20) Zheng, Y.; Liu, H.; Wang, Y.; Zhu, C.; Wang, S.; Cao, J.; Zhu, S. Accumulating Microparticles and Direct-Writing Micropatterns Using a Continuous-Wave Laser-Induced Vapor Bubble. Lab Chip 2011, 11, $3816-3820$

(21) Rajeeva, B. B.; Kunal, P.; Kollipara, P. S.; Acharya, P. V.; Joe, M.; Ide, M. S.; Jarvis, K.; Liu, Y.; Bahadur, V.; Humphrey, S. M.; Zheng, Y. Accumulation-Driven Unified Spatiotemporal Synthesis and Structuring of Immiscible Metallic Nanoalloys. Matter 2019, 1, 16061617.

(22) Fujii, S.; Fukano, R.; Hayami, Y.; Ozawa, H.; Muneyuki, E.; Kitamura, N.; Haga, M.-a. Simultaneous Formation and Spatial Patterning of $\mathrm{ZnO}$ on ITO Surfaces by Local Laser-Induced Generation of Microbubbles in Aqueous Solutions of [ $\mathrm{Zn}(\mathrm{NH} 3) 4]$ 2+. ACS Appl. Mater. Interfaces 2017, 9, 8413-8419.

(23) Jones, S.; Andrén, D.; Antosiewicz, T. J.; Käll, M. Ultrafast Modulation of Thermoplasmonic Nanobubbles in Water. Nano Lett. 2019, 19, 8294-8302.

(24) Jones, P. H.; Marago, O. M.; Volpe, G. Optical Tweezers Principles and Applications, 1st ed.; Cambridge University Press: Cambridge, 2015.

(25) Vaught, J. L.; Cloutier, F. L.; Donald, D. K.; Meyer, J. D.; Tacklind, C. A.; Taub, H. H. Thermal Inkjet Printer. US4490728A, 1984-12-25.

(26) Wang, Y.; Zaytsev, M. E.; Lajoinie, G.; Le-The, H.; Eijkel, J. C. T.; van den Berg, A.; Versluis, M.; Weckhuysen, B. M.; Zhang, X.; Zandvliet, H. J. W.; Lohse, D. Giant and Explosive Plasmonic Bubbles by Delayed Nucleation. Proc. Natl. Acad. Sci. U. S. A. 2018, 115, $7676-7681$.

(27) Namura, K.; Okai, S.; Kumar, S.; Nakajima, K.; Suzuki, M. SelfOscillation of Locally Heated Water Vapor Microbubbles in Degassed Water. Adv. Mater. Interfaces 2020, 7, 2000483-10.

(28) Namura, K.; Nakajima, K.; Kimura, K.; Suzuki, M. Photothermally Controlled Marangoni Flow around a Micro Bubble. Appl. Phys. Lett. 2015, 106, 043101-043105. 
(29) Namura, K.; Nakajima, K.; Suzuki, M. Investigation of Transition from Thermal- to Solutal-Marangoni Flow in Dilute Alcohol/Water Mixtures Using Nano-Plasmonic Heaters. Nanotechnology 2018, 29, 065201-065207.

(30) Johnson, P. B.; Christy, R. W. Optical Constants of the Noble Metals. Phys. Rev. B 1972, 6, 4370-4379. 